International Journal of Antennas (JANT) Vol.2, No.1, January 2016

\title{
PRELIMINARY DESIGN AND CHARACTERISATION OF A NOVEL T-SHAPED NANO-ANTENNA ON DIAMOND LIKE CARBON MATERIAL
}

\author{
Ahmad Bahar ${ }^{1}$, Mohamed Ismaeel Maricar ${ }^{2}$, Richard $^{3}$ \\ Cross Emerging Technologies Research Centre \\ De Montfort University Leicester,UK
}

\begin{abstract}
The rapid growth of nanotechnology has led to the development of many devices with advanced characteristics such as high frequencies Gunn diodes, transistors, nano-antennas etc. Nano-antenna applications are numerous and encompass a variety of fields such as broadband communications, imaging, sensing, energy harvesting, and disease diagnosis. In this research paper, A novel T-shaped nano-antenna was designed and its resonant frequency was analysed. Moreover, parametric investigation had been performed to figure out the effects of T-shaped nano-antenna on Diamond Like Carbon (DLC) material. The momentum model in Advanced Design System (ADS) software was used to simulate the novel T-shaped nano-antenna and the results were directly compared to other nano-antennas. Initial results suggests that the T-shaped nano-antenna had a higher bandwidth and smaller geometrical size when compared to the other nano-antenna at Terahertz frequency.
\end{abstract}

\section{Index Terms}

T-shaped antenna, resonant frequency, diamond like carbon material and bandwidth.

\section{INTRODUCTION}

Antennas are widely used in low frequency part of the electromagnetic spectrum, but recently nano-antennas have been explored up to visible range [1][2][3]. There is a wide range of antenna forms that are able to accommodate the stated operating frequency or may be scaled correspondingly for recommended operation.[4][5][6][7].

On the other hand, a greater number of designs feature a wide surface space which may not proper for suitable applications, while a few designs may not be appropriate because of the bandwidth limitations. [8]. Accordingly, the main objective for the present design was to resolve a compact nano-antenna which can provide the recommended impedance and gain achievement over frequency $\left[\begin{array}{ll}1 \mathrm{THz}-5 & \mathrm{THz}\end{array}\right]$. 
The design of an antenna working at optical frequencies is not a straightforward task, due to the inherent nature of the optical radiation and to the dispersive properties of the materials in such a frequency range. Following the previous works by Alu' and Engheta [9][10][11], from an electrical engineering perspective, a possible way to design nano-antennas consists in adapting the concepts commonly used at radio and microwave frequencies to the design of optical nano-antennas. Such an approach has been successfully employed in the case of simple nano-antennas,such as the nano-dipoles and nanodimers [9][10][11], this is also applicable for more complex radiators, such as the bowtie[12], the spiral [13], and the Yagi-Uda[14] antennas.

The concept of the design was to realise a radiating device which is able to generate several resonances in the operating spectrum to obtain ultra-wideband performance. The proposed nanoantenna has a novel T-shaped that is capable of preserving a low profile and fitting in a tiny area and gaining resonances for wideband performance at the same time. The following stage of the design was to focus on further improvements for the overall performance. Section III will focus on the major steps for enhancing the propsed T-shaped nano-antenna on DLC substance by utilising momentum model in Advanced Design System (ADS)[15]. The structure was designed to achieve the circular polarisation in terahertz frequencies. The novel T-shaped nanoantenna was found to have higher bandwidth and return loss when compared to the other nanoantennas[16][17][18].

\section{NANO-ANTENNA BACKGROUND}

Antennas are vital devices in modern day communications. The first era of antennas began from the first test of an antenna done by Marconi in 1897. He sent the world's first ever wireless communication over Bristol Channel and sent a Morse code from Flat Holm Island to Lavernock Point in Penarth (a distance of 3.7 miles).

Antennas at that time were wires which were used for transmitting and receiving radio waves. Later in 1992 [19],researchers improved the design of microwaves antennas which facilitated the production of a variety of new structures (e.g. microstrips, apertures, phased-array radars)[20]) which had reduced size as this depends on the wavelength of the frequency in question.

The normal size of antennas working in radio and microwave frequencies starts from a hundred metres to a few millimetres[21]. Shrinking antennas below this range (ie. into the nanoscale) is the area of focus for this work. Consequently, these devices operate in the optical regime are also known as optical antennas.

Until the tools and methods were developed to fabricate devices at the nanoscale scale (largely within the semiconductor industry), it is only now that the capabilities and potential applications of nanoantennas can be truly explored [22]. Nanotechnology can be defined as the utilisation of scientific knowledge to design, fabricate, control and use matter at the nanometer scale (about $1100 \mathrm{~nm}$ ). At this scale size, different characteristics and phenomena can emerge[23] and it is also applicable to microwave antennas to some extent. 


\section{DESIGN AND ANALYSIS OF A T-SHAPED NANOANTENNAS}

A schematic view of the T-shaped nano-antenna is illustrated in figure 1 and 2 . The nano-antenna has three layers; ground plate, dielectric medium, and the metallisation layer. The metallisation layer has a T-shaped with an inner length of $\mathrm{L}$, thickness of $\mathrm{T}$ with a sectorial angle $\theta$, the apex corner of the nano-antenna is straight fed from a $50 \mathrm{Ohm}$ micro-strip line.

The structure of the nano-antenna can be fabricated on a dielectric substrate with a thickness of $\mathrm{H}$ and a relative permittivity $s r$. Using the electromagnetic package in ADS, the structure design was analysed by setting it up as a momentum model. Special care was conducted in choosing the mesh size to obtain sensible results and it is one of the specification for the high frequency antenna. In practise, the mesh size was limited by the available desktop computer. The nanoantennas were analysed on DLC material with a thickness of $100 \mathrm{~nm}$ and a relative permittivity of 2.615. The $50 \Omega$ microstrip line had a width $\mathrm{W}$ of $60 \mathrm{~nm}$ and these were calculated by using the

ADS (line calculator). It was founded that by reducing the inner length $\mathrm{L}$ of the T-shaped nanoantenna, the resonant frequency was increased. The T-shaped nano antennas had superior performance over the bowtie antenna and it had a higher Q. In practical terms, it is difficult to calculate the length of a T-shaped nano antenna for a particular resonant frequency. Separate work was carried out and polynomial equations relating to radius and internal angle for the nano antennas were obtained. The simulated result for a T-shaped nano antenna having an inner length of $400 \mathrm{~nm}$ and sectorial angle of 60 degree is shown in figure 3 . The simulated results show that the T-shaped antenna was resonating at $1.3 \mathrm{THz}$ and $3.3 \mathrm{THz}$ with a operational bandwidth of more than $100 \%$ performs significantly better than the traditional nano-antenna. Simulated gain and directivity performance for T-shaped nano antenna is shown in figure 4, which shows that a gain of $1.8 \mathrm{dBi}$ at $1.3 \mathrm{THz}$ and $1.1 \mathrm{dBi}$ at $3.3 \mathrm{THz}$ and it has almost flat response thought out the majority of the frequency. A typical radiation pattern for the novel $\mathrm{T}$-shaped nano-antenna is shown in figure 5 . The antennas were designed to radiate in broad side i.e. perpendicular to the axis of the patch. The main beam is sharp between $90^{\circ}$ and $270^{\circ}$ while minimum in between $0^{\circ}$ and $180^{\circ}$. Figure 6 verifies the designed antennas directivity. Figure 7 shows even more precise graph of radiation pattern shown by Electro Magnetic Design Solver, which is an extension of ADS.

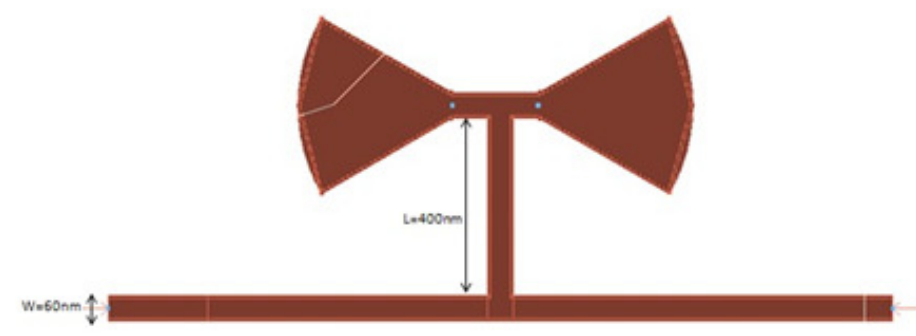

Fig. 1. Schematic view of the T-shaped nano-antennas (top-view) 


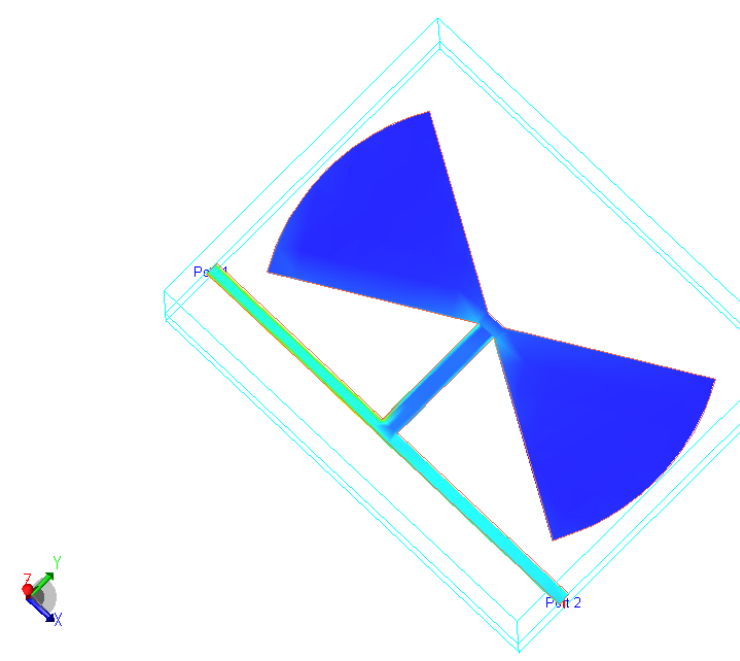

Fig. 2. Schematic view of the T-shaped nano-antennas (angular-view)

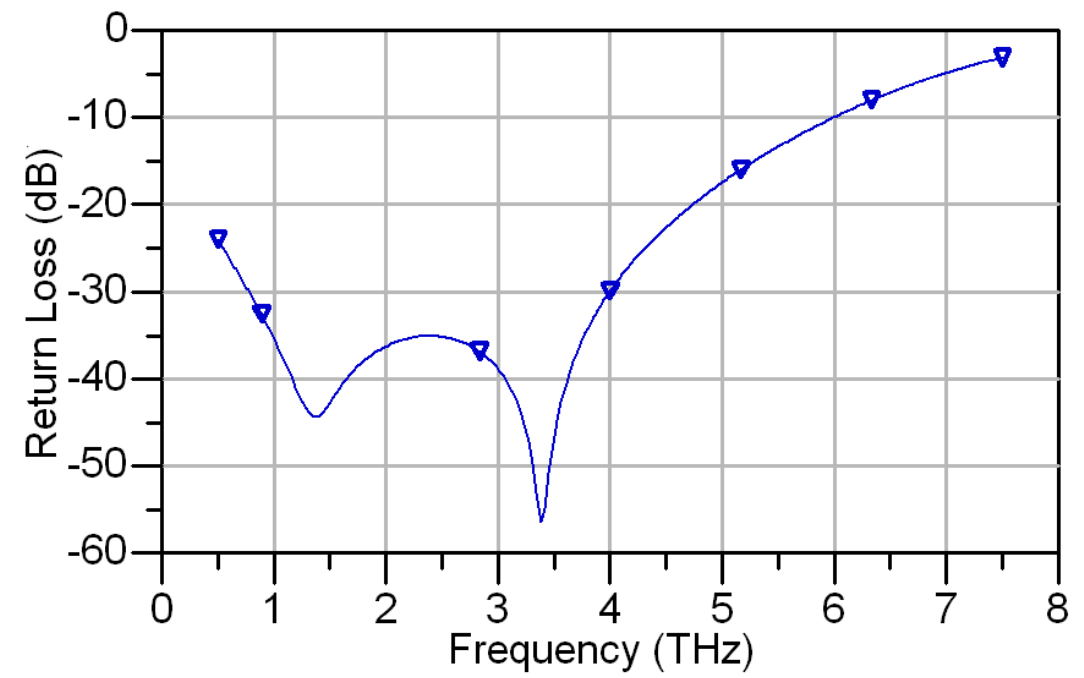

Fig. 3. Simulated return loss response of the designed T-Shaped nano-antennas 
International Journal of Antennas (JANT) Vol.2, No.1, January 2016

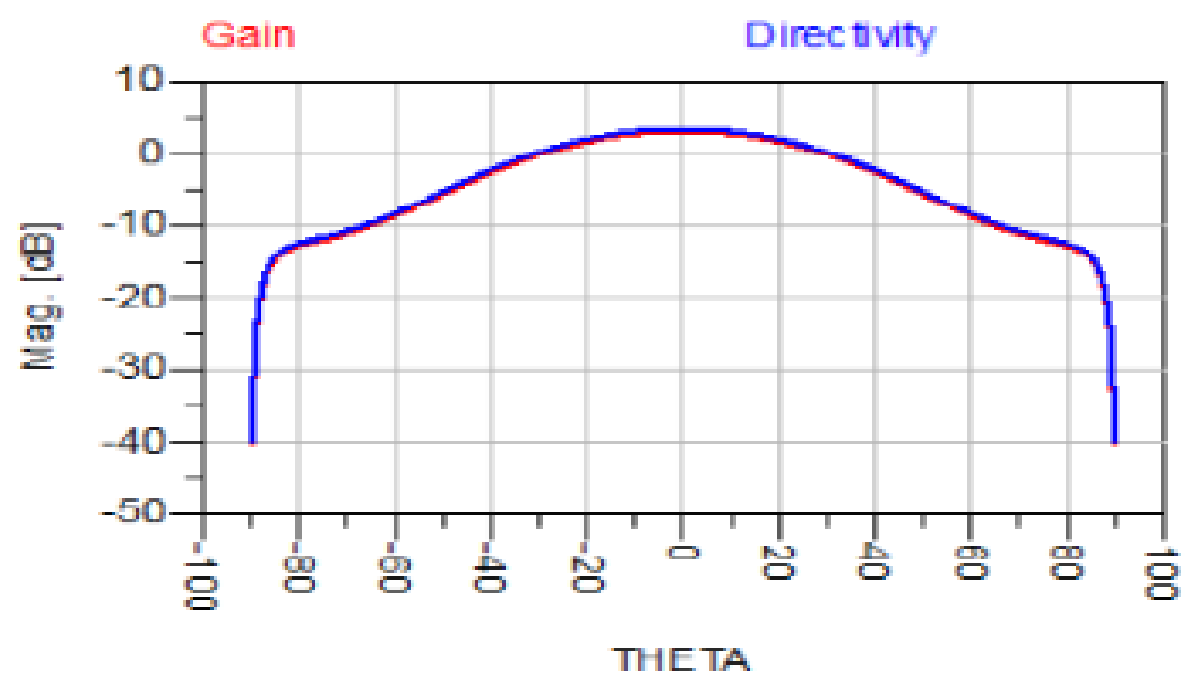

Fig. 4. Gain and directivity of the T-shaped nano-antenna

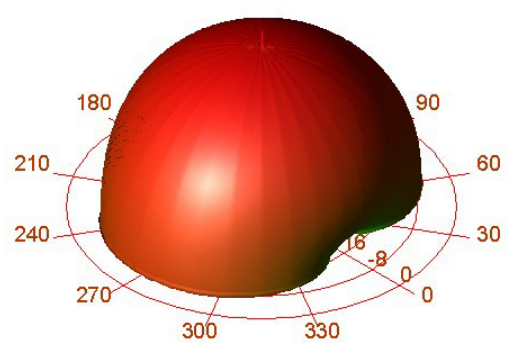

Fig. 5. Front view of 3D directivity pattern 


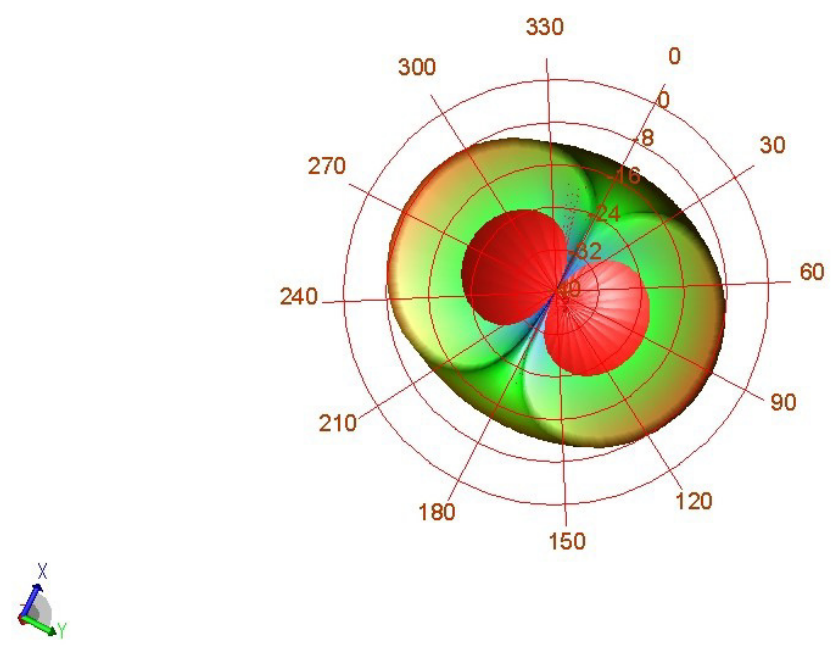

Fig. 6. Opposite view of 3D directivity pattern

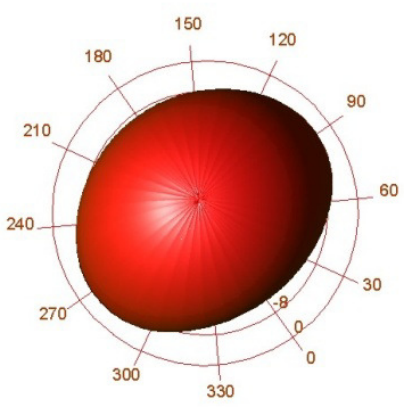

\section{CONCLUSION}

Fig. 7. 3D Radiation Pattern in EMDS

This paper describes a novel T-shaped nano-antenna on DLC material. The novel T-shaped nano-antenna was designed and analysed by using momentum model in ADS software and the simulations results were directly compared with other nano-antennas. The results indicate that the novel T-shaped nano-antenna had a smaller physical size and higher bandwidth when compared to the other nano-antennas at terahertz frequency. 
International Journal of Antennas (JANT) Vol.2, No.1, January 2016

\section{REFERENCES}

[1] Javad N Farahani, Hans-Ju“rgen Eisler, Dieter W Pohl, Michae“1 Pavius, Philippe Flu“ckiger, Philippe Gasser, and Bert Hecht. Bow-tie optical antenna probes for single-emitter scanning near-field optical microscopy. Nanotechnology, 18(12):125506, 2007.

[2] Alberto G Curto, Giorgio Volpe, Tim H Taminiau, Mark P Kreuzer, Romain Quidant, and Niek F van Hulst. Unidirectional emission of a quantum dot coupled to a nanoantenna. Science, 329(5994):930 933, 2010.

[3] TH Taminiau, FD Stefani, FB Segerink, and NF Van Hulst. Optical antennas direct single-molecule emission. Nature Photonics, 2(4):234- 237, 2008.

[4] AA Lestari, AG Yarovoy, and LP Ligthart. An efficient ultra-wideband bow-tie antenna. In Microwave Conference, 2001. 31st European, pages 1-4. IEEE, 2001.

[5] Ben Allen, Mischa Dohler, Ernest Okon, Wasim Malik, Anthony Brown, and David Edwards. Ultra wideband antennas and propagation for communications, radar and imaging. John Wiley \& Sons, 2006.

[6] Yan-Lin Chen, Cheng-Li Ruan, and Lin Peng. A novel ultra-wideband bow-tie slot antenna in wireless communication systems. Progress In Electromagnetics Research Letters, 1:101-108, 2008.

[7] Katsuki Kiminami, Akimasa Hirata, and Toshiyuki Shiozawa. Double-sided printed bow-tie antenna for uwb communications. IEEE Antennas and Wireless Propagation Letters, 1(3):152-153, 2004.

[8] Manas Sarkar, Santanu Dwari, and Anitha Daniel. Compact printed monopole antenna for ultrawideband application with dual band- notched characteristic. Microwave and Optical Technology Letters, 55(11):2595-2600, 2013.

[9] Andrea Alu and Nader Engheta. Tuning the scattering response of optical nanoantennas with nanocircuit loads. Nature photonics, 2(5):307-310, 2008.

[10] Andrea Alu' and Nader Engheta. Input impedance, nanocircuit loading, and radiation tuning of optical nanoantennas. Physical review letters, 101(4):043901, 2008.

[11] Andrea Alu' and Nader Engheta. A hertzian plasmonic nanodimer as an efficient optical nanoantenna. arXiv preprint arXiv:0807.1783, 2008.

[12] Hongcang Guo, Todd P Meyrath, Thomas Zentgraf, Na Liu, Liwei Fu, Heinz Schweizer, and Harald Giessen. Optical resonances of bowtie slot antennas and their geometry and material dependence. Optics express, 16(11):7756-7766, 2008.

[13] Ranjan Singh, Carsten Rockstuhl, Christoph Menzel, Todd P Meyrath, Mingxia He, Harald Giessen, Falk Lederer, and Weili Zhang.Spiral-type terahertz antennas and the manifestation of the mushiake principle. Optics express, 17(12):9971-9980, 2009.

[14] Davide Ramaccia, Filiberto Bilotti, Alessandro Toscano, Alessandro Massaro, and Roberto Cingolani. Electrical and radiation properties of a horn nano-antenna at near infrared frequencies. In Antennas and Propagation (APSURSI), 2011 IEEE International Symposium on, pages 2407-2410. IEEE, 2011.

[15] Agilent. Advanced Design System (ADS) Tutorial, 2009.

[16] Shailesh Raut and Aldo Petosa. A compact printed bowtie antenna for ultra-wideband applications. In Microwave Conference, 2009.EuMC 2009. European, pages 081-084. IEEE, 2009.

[17] HaiWen Liu, Hao Jiang, Xuehui Guan, JiuHuai Lei, and Shen Li. Single-feed slotted bowtie antenna for triband applications. Antennas and Wireless Propagation Letters, IEEE, 12:1658-1661, 2013.

[18] A Thabet, Adel Z El Dein, and A Hassan. Design of compact microstrip bandpass filter by using new nano-composite materials. In Nanoelectronics Conference (INEC), 2011 IEEE 4th International, pages 1-2. IEEE, 2011.

[19] David M Pozar and Daniel H Schaubert. Microstrip antennas: the analysis and design of microstrip antennas and arrays. John Wiley \& Sons, 1995.

[20] Constantine A Balanis. Antenna theory: analysis and design. John Wiley \& Sons, 2012.

[21] Q-Han Park. Optical antennas and plasmonics. Contemporary Physics, 50(2):407-423, 2009.

[22] Palash Bharadwaj, Bradley Deutsch, and Lukas Novotny. Optical antennas. Advances in Optics and Photonics, 1(3):438-483, 2009. 
International Journal of Antennas (JANT) Vol.2, No.1, January 2016

[23] Thomas P.J. Linsinger, Gert Roebben, Conxita Solans, and Roland Ramsch. Reference materials for measuring the size of nanoparticles.TrAC Trends in Analytical Chemistry, 30(1):18 - 27, 2011. Characterization, Analysis and Risks of Nanomaterials in Environmental and Food Samples. 